\title{
Genetic evidence for contribution of human dispersal to the genetic diversity of EBA-175 in Plasmodium falciparum
}

\author{
Yoshiki Yasukochi ${ }^{1 *}$, Izumi Naka ${ }^{1,2}$, Jintana Patarapotikul ${ }^{3}$, Hathairad Hananantachai ${ }^{4}$ and Jun Ohashi ${ }^{1,2}$
}

\begin{abstract}
Background: The 175-kDa erythrocyte binding antigen (EBA-175) of Plasmodium falciparum plays a crucial role in merozoite invasion into human erythrocytes. EBA-175 is believed to have been under diversifying selection; however, there have been no studies investigating the effect of dispersal of humans out of Africa on the genetic variation of EBA-175 in P. falciparum.

Methods: The PCR-direct sequencing was performed for a part of the eba-175 gene (regions II and III) using DNA samples obtained from Thai patients infected with P. falciparum. The divergence times for the P. falciparum eba-175 alleles were estimated assuming that $P$. falciparum/Plasmodium reichenowi divergence occurred 6 million years ago (MYA). To examine the possibility of diversifying selection, nonsynonymous and synonymous substitution rates for Plasmodium species were also estimated.

Results: A total of 32 eba-175 alleles were identified from 131 Thai P. falciparum isolates. Their estimated divergence time was 0.13-0.14 MYA, before the exodus of humans from Africa. A phylogenetic tree for a large sequence dataset of $P$. falciparum eba-175 alleles from across the world showed the presence of a basal Asian-specific cluster for all $P$. falciparum sequences. A markedly more nonsynonymous substitutions than synonymous substitutions in region II in P. falciparum was also detected, but not within Plasmodium species parasitizing African apes, suggesting that diversifying selection has acted specifically on P. falciparum eba-175.

Conclusions: Plasmodium falciparum eba-175 genetic diversity appeared to increase following the exodus of Asian ancestors from Africa. Diversifying selection may have played an important role in the diversification of eba-175 allelic lineages. The present results suggest that the dispersals of humans out of Africa influenced significantly the molecular evolution of $P$. falciparum EBA-175.
\end{abstract}

Keywords: Diversifying selection, EBA-175, Human evolution, Out-of-Africa, Plasmodium falciparum

\section{Background}

Approximately 200 Plasmodium species parasitize different vertebrate groups, including lizards, birds, and mammals [1]. Of them, five Plasmodium species, namely Plasmodium falciparum, Plasmodium vivax, Plasmodium ovale (consisting of two subspecies, Plasmodium ovale curtisi and Plasmodium ovale wallikeri [2]), Plasmodium malariae, and Plasmodium knowlesi, can

\footnotetext{
*Correspondence: hyasukou@proof.ocn.ne.jp

${ }^{1}$ Department of Biological Sciences, Graduate School of Science, The University of Tokyo, 7-3-1, Hongo, Bunkyo-ku, Tokyo 113-0033, Japan Full list of author information is available at the end of the article
}

infect humans. In particular, $P$. falciparum causes the most severe form of malaria in humans; however, when compared with Plasmodium reichenowi found in chimpanzees (Pan troglodytes), P. falciparum appears to be phylogenetically distantly related to other human malaria parasites [1, 3-5].

The 175-kDa erythrocyte binding antigen (EBA-175) of $P$. falciparum binds with a sialic acid on human glycophorin A (GYPA); the interaction of these molecules is the part of a major pathway for malaria parasite invasion into erythrocytes. The primary structure of $e b a-175$ gene is divided into seven regions, region I-VII [6]. Region II 
is a cysteine-rich region responsible for the interaction between EBA-175 and GYPA on the erythrocyte surface [7]. Previous studies have shown a significant excess of nonsynonymous substitutions over synonymous substitutions in this region in P. falciparum [8-11]; however, no studies have assessed to date the ratio of nonsynonymous to synonymous substitutions in other closely related Plasmodium species. Nonsynonymous substitution rates of host and pathogen are expected to increase synergistically as a consequence of the host-pathogen co-evolutionary arms race. When human and macaque genomes were compared, the gypa gene shows the highest number of nonsynonymous substitutions per nonsynonymous sites among 280 genes [10]. In addition, the rate of nonsynonymous substitutions in eba-175 gene is also the highest among 10 genes compared between $P$. falciparum and $P$. reichenowi [10]. Thus, the high level of genetic polymorphisms shown by eba-175 and gypa is likely to result from host-pathogen coevolution.

Amino acid substitutions in region II of $e b a-175$ and gypa seem to have been enhanced by diversifying selection. However, it remains unclear how human evolution has influenced $P$. falciparum eba-175 genetic diversity. To elucidate this, in the present study, the regions II and III of eba-175 from a Thai P. falciparum population were examined. When compared with $P$. reichenowi eba-175 sequence, eba-175 alleles from this population can be traced back to approximately 130-140 thousand years ago. A further analysis for a large sequence dataset of $P$. falciparum eba-175 alleles from across the world revealed an Asian-specific basal cluster in a phylogenetic tree. In addition, an evolutionary analysis detected a significant excess of nonsynonymous substitutions over synonymous substitutions in the region II of $e b a-175$ within the Thai $P$. falciparum population, but not within Plasmodium populations parasitizing African apes. Thus, the results from the present study suggest that human dispersal out of Africa contributed to the genetic diversification of P. falciparum eba-175.

\section{Methods}

\section{Ethics statement}

This study was approved by the Institutional Review Board of the Faculty of Tropical Medicine, Mahidol University, and the Research Ethics Committee of the School of Medicine, The University of Tokyo.

\section{Subjects}

Peripheral blood samples were obtained from $203 P$. falciparum-infected patients from Thailand. In addition, a dataset of nucleotide sequences including isolates of human P. falciparum, gorilla (Gorilla gorilla)
Plasmodium, chimpanzee Plasmodium, and P. reichenowi was built from data obtained from [12].

\section{Experimental procedures for DNA sequencing of $e b a-175$}

Genomic DNA was extracted from pretreated peripheral blood samples from patients infected with $P$. falciparum using a QIAamp Blood Kit (Qiagen, Hilden, Germany). DNA fragments covering a part of the eba175 coding sequence (regions II and III) of $P$. falciparum were amplified by PCR using the following sets of primers: EBA175-fragment1, 5'-ggaagaaatacttcatctaataacg-3' (forward) and $5^{\prime}$-catcctttacttctggacacatcg- $3^{\prime}$ (reverse), and EBA175-fragment2, 5'-gagactctgaaggttgaatgcaa- $3^{\prime}$ (forward) and $5^{\prime}$-aggtgtattagacatatcttggtc- $3^{\prime}$ (reverse). These primers were designed based on the eba-175 reference sequence from $P$. falciparum (GenBank accession no. X52524). PCR amplification was performed in a $13.0-\mu \mathrm{L}$ reaction mixture containing $0.125 \mu \mathrm{L}(0.125 \mu \mathrm{M})$ each of forward and reverse primers, $0.125 \mu \mathrm{L}$ TAKARA LA Taq ${ }^{\mathrm{TM}}$ (5 units/ $\mu \mathrm{L}$ ), $1.25 \mu \mathrm{L} 10 \times \mathrm{LA} \mathrm{PCR}^{\mathrm{TM}}$ Buffer II $\left(\mathrm{Mg}^{2+}\right.$ free), $1.25 \mu \mathrm{L} 25 \mathrm{mM} \mathrm{MgCl}, 1.25 \mu \mathrm{L} 2.5 \mathrm{mM}$ dNTP mixture, $0.5 \mu \mathrm{L}$ ( $5 \mathrm{ng}$ ) of genomic DNA template, and $8.375 \mu \mathrm{L} \mathrm{dH}_{2} \mathrm{O}$ using a GeneAmp ${ }^{\circledR}$ PCR System 9700 (Applied Biosystems, Foster City, CA, USA). The PCR cycling conditions for each primer pair were $60 \mathrm{~s}$ initial denaturation at $94^{\circ} \mathrm{C}$, followed by 40 cycles of $30 \mathrm{~s}$ denaturation at $94^{\circ} \mathrm{C}, 30 \mathrm{~s}$ annealing at $56^{\circ} \mathrm{C}$, and $150 \mathrm{~s}$ extension at $72^{\circ} \mathrm{C}$, and a final step of $5 \mathrm{~min}$ extension at $72^{\circ} \mathrm{C}$. The PCR products were subsequently sequenced using an ABI Prism 3100 Genetic Analyzer (Applied Biosystems, Foster City, CA, USA). Primer sequences used for direct sequencing are available upon request. The isolates showing multiple superimposed electropherogram peaks at a single site following PCR-direct sequencing and a secondary peak greater than $30 \%$ of the predominant peak were considered to be mixed infections and excluded from further analyses. Low-quality sequences (i.e., high background noise or too weak signal) were also excluded. As a result, 131 sequences representing the single or most abundant sequence in each DNA sample were included in the analyses.

\section{Data analyses}

The nucleotide sequences obtained were aligned and translated into putative amino acid sequences using MEGA v.5.2 [13]. To examine the phylogenetic relations among 32 distinct eba-175 Thai $P$. falciparum alleles and two eba-175 P. reichenowi alleles (CBXM000000000 and AJ251848), a maximum likelihood (ML) tree was constructed based on the Hasegawa-Kishino-Yano model [14]. To obtain the ML tree, a nearest-neighbor-interchange (NNI) search was applied. In addition, a neighborjoining (NJ) [15] tree was generated using 194 eba-175 
partial region II sequences from $P$. falciparum worldwide and two P. reichenowi sequences (CBXM000000000 and AJ251848), based on the Nei-Gojobori (NG) model [16] and the Jukes-Cantor (JC) correction [17]. The construction of phylogenetic trees and estimation of best-fit substitution model for the ML tree were implemented in MEGA v.5.2 [13]. All the positions containing insertions/ deletions were eliminated from the analyses (complete deletion option). Branch support values were computed by bootstrap analyses with 1,000 replications. A network of 194 P. falciparum and two $P$. reichenowi eba-175 alleles was also constructed based on synonymous substitutions using the neighbor-net method [18] in SplitsTree4 ver. 4.13.1 [19].

The time to the most recent common ancestor (tMRCA) of the eba-175 alleles from Thai falciparum was estimated from the linearized tree based on synonymous substitutions among the 32 distinct eba-175 alleles by using the MEGA v5.2 [13]. The neutral substitution rate was calculated assuming that $P$. falciparum and $P$. reichenowi diverged 6 million years ago (MYA) $[5,20]$. In addition, the MCMCTree program in the PAML 4.8 package [21] was used to estimate tMRCA based on the amino acid sequences. The minimum and maximum age constraints on the root age (the divergence time between $P$. falciparum and P. reichenowi) were set to 5 and 7 MYA, respectively. The tMRCA estimation was based on a WAG model [22] for amino acid substitutions. In the MCMC process, sampling occurred every 100 generations for 10,000 generations and the first 50,000 generations were discarded as burn-in.

To detect the signatures of natural selection, the number of nonsynonymous substitutions per nonsynonymous site $\left(d_{\mathrm{N}}\right)$ and synonymous substitutions per synonymous site $\left(d_{S}\right)$ for all the pairs formed by the 32 distinct Thai P. falciparum alleles, 16 from chimpanzee Plasmodium spp., 11 from gorilla Plasmodium spp., and four from $P$. reichenowi sequences were estimated using the NG model with the JC correction in MEGA v.5.2 [13]. Significant difference between $d_{\mathrm{N}}$ and $d_{\mathrm{S}}$ was assessed by Wilcoxon signed-rank test. For all the 131 Thai $P$. falciparum isolates, the numbers of nonsynonymous substitutions per nonsynonymous site $\left(\pi_{\mathrm{N}}\right)$ and synonymous substitutions per synonymous site $\left(\pi_{S}\right)$ were also calculated in the same manner as $d_{\mathrm{N}}$ and $d_{\mathrm{S}}$. In addition, the McDonald-Kreitman (MK) test [23] was performed for detecting natural selection signal using DnaSP v5 software [24]. Tajima's $D$ test [25] was performed for 131 Thai $P$. falciparum eba-175 sequences using DnaSP v5 software [24], where the test statistic was analytically calculated. A twosided $P$ value of less than 0.05 was considered statistically significant.
A Wu-Kabat plot was used to estimate the level of amino acid variability for the 32 distinct Thai $P$. falciparum eba-175 alleles [26]. The Wu-Kabat plot estimates the level of variability for each amino acid position in the sequence alignment, measured as the number of amino acids at each site divided by the maximum frequency of amino acid for all sites.

\section{Results}

\section{Detection of eba- 175 alleles}

The nucleotide sequences of regions II and III of $P$. falciparum eba-175 were obtained by PCR-direct sequencing. The eba-175 region III showed highly divergent dimorphic segments, the F and $\mathrm{C}$ segments [termed Fseg (423 bp) and Cseg (342 bp)], as reported by Ware et al. [27]. A total of 32 distinct alleles [20 Fseg alleles (ca. 2,740 bp) and 12 Cseg alleles (ca. 2,660 bp)], defined by 30 polymorphic sites including insertions/deletions (site 744-749, and 2094), were detected from 131 P. falciparum isolates from Thailand [Fig. 1; Fseg alleles: F1_1-F20 (Genbank accession numbers LC008232-LC008251) and Cseg alleles: C1-C12 (Genbank accession numbers LC008252-LC008263)]. The nucleotide sequences of the 32 alleles were translated into 31 distinct amino acid sequences. Synonymous substitutions were found at only two sites in the allele sequences. On the other hand, nonsynonymous substitutions were found at 21 sites, not including an insertion/deletion site. The $\mathrm{F}$ and $\mathrm{C}$ segments were excluded from further analyses.

\section{Phylogenetic relations among eba-175 alleles}

A ML tree was constructed based on the nucleotide sequences of 32 eba-175 alleles from Thai $P$. falciparum, and two eba-175 alleles from P. reichenowi (Fig. 2). In this ML tree, Fseg alleles formed a monophyletic clade with a relatively low bootstrap value (51\%). A NJ tree was generated based on synonymous substitutions in region II to further analyze the phylogenetic relations among 194 $P$. falciparum eba-175 alleles, including a large sequence dataset from Genbank [12] (Fig. 3). In particular, in this NJ tree, some P. falciparum alleles isolated from Asia formed a single clade rooted in the most basal node from where all $P$. falciparum sequences diverged. All the alleles included in the Asian clade were characterized by a mutation in site 441, as shown in Fig. 1 (Asian-specific alleles contain cytosine nucleotide at site 441 , where all other sequences contain a thymine). This Asian-specific cluster was also supported by the neighbor-net method (Additional file 1) and suggests that an ancestral allele in the cluster emerged in Asia and then rapidly spread across Asia after the out-of-Africa. 


\begin{tabular}{|c|c|c|c|c|c|c|c|c|c|c|c|c|c|c|c|c|c|c|c|c|c|c|c|c|c|c|c|}
\hline \multirow[t]{2}{*}{ Allele } & \multicolumn{27}{|c|}{ Mutation site } \\
\hline & & & & & & & & & & & & & & & & & & & 1 & 1 & 1 & 11 & 11 & 12 & 22 & & \\
\hline & 3 & 33 & 33 & 45 & 57 & 77 & 7 & 7 & 7 & 7 & 7 & 7 & 7 & 7 & 7 & & 9 & 2 & 2 & 2 & 3 & 56 & 68 & 80 & 3 & & 0 \\
\hline & 6 & 67 & 79 & 44 & 40 & 01 & 4 & 4 & 4 & 4 & 4 & 4 & 5 & 5 & 5 & 7 & 8 & 7 & 7 & 9 & 1 & $3 \varepsilon$ & 84 & 49 & 3 & 5 & 5 \\
\hline & 3 & 78 & 89 & 19 & 97 & 71 & 4 & 5 & 6 & 7 & 8 & 9 & 0 & 5 & 7 & 7 & 5 & 4 & 9 & 3 & 8 & 35 & 96 & 64 & 7 & 0 & 8 \\
\hline$F 1-1$ & $A$ & $T G$ & G G & $T G$ & G A & $A C$ & $A$ & $\mathrm{~T}$ & $\mathrm{~T}$ & $\mathrm{~T}$ & C & $A$ & G & C & A & A & A & $\mathrm{T}$ & $\mathrm{T}$ & G & $\mathrm{C}$ & A $A$ & $A \mathrm{~A}$ & A $T$ & A & & $\mathrm{T}$ \\
\hline$F 1-2$ & . & . . & . . & . & . . & . . & . & . & . & . & . & . & . & . & & & & & . & . & . & . & . . & . $\mathrm{C}$ & $=$. & . & . \\
\hline F3 & . & . &. & . & . . & . . & . & . & . & . & . & . & . & . & . & & & & . & . & . & & G. & . $\mathrm{C}$ & {$[$.} & . & . \\
\hline$F 4$ & G & . & . $A$ & . & . $\mathrm{T}$ & $\mathrm{T} T$ & - & - & - & - & - & - & A & A & $\mathrm{T}$ & & & & . & . & $A$ & . &. $\mathrm{G}$ & $G C$ & {$[$.} & . & . \\
\hline F5 & . & . &. & . & . & . $\mathrm{T}$ & - & - & - & - & - & - & A & A & $\mathrm{T}$ & & . & A & C & . & . & . &.$G$ & $\mathrm{G} C$ & {$[$.} & . & . \\
\hline F6 & . & . & . $A$ & . & . $\mathrm{T}$ & $\mathrm{T} T$ & . & . & . & . & . & . & $A$ & . & & & & & . & . & . & . & & $\mathrm{GC}$ & {$[$.} & . & . \\
\hline$F 7$ & . & . & . $A$ & . & . . & . . & . & . & . & . & . & . & . & . & . & & & & . & . & . & . & & $\mathrm{GCC}$ & {$[$.} & . & . \\
\hline F8 & . & . & . . & . & . & . & . & . & . & . & . & . & . & . & $\cdot$ & . & . & & . & . & . & . &.$G$ & $\mathrm{GCC}$ & $E$. & . & . \\
\hline F9 & . & . & . & . & . $\mathrm{T}$ & $\mathrm{T}$. & - & - & - & - & - & - & A & A & $\mathrm{T}$ & & & & . & . & . & . &.$G$ & $\mathrm{G} C$ & {$[$.} & . & . \\
\hline F10 & . & . & . & . & . & . $\mathrm{T}$ & - & - & - & - & - & - & A & A & $\mathrm{T}$ & $\cdot$ & & & . & . & . & . &.$G$ & $\mathrm{G} C$ & {$[$.} & . & . \\
\hline F11 & G & A. & . $A$ & . & $\mathrm{T}$ & $\mathrm{T} T$ & - & - & - & - & - & - & A & A & $\mathrm{T}$ & . & & & . & . & . & . &.$G$ & $\mathrm{GCC}$ & $E$. & . & . \\
\hline F12 & G & . . & . $A$ & . & $\mathrm{T}$ & $\mathrm{T} T$ & - & - & - & - & - & - & $A$ & A & $\mathrm{T}$ & & . & & . & . & . & . & . G & $\mathrm{GC}$ & {$[$.} & . & . \\
\hline F13 & . & . & . & . & $\mathrm{T}$ & $\mathrm{T} T$ & - & - & - & - & - & - & $A$ & A & $\mathrm{T}$ & . & . & & . & . & . & . & . G & $\mathrm{GC}$ & {$[$.} & . & . \\
\hline F14 & . & . & . & . & . & $\mathrm{T} T$ & - & - & - & - & - & - & $A$ & A & $\mathrm{T}$ & & $\mathrm{T}$ & & . & . & . & . & . G & $\mathrm{GC}$ & {$[$.} & . & . \\
\hline F15 & $\mathrm{G}$ & . $\mathrm{A}$ & $A$ & . & . & . . & . & . & . & . & . & . & . & . & . & & & & . & C & $A$ & C. & . G & $\mathrm{GCC}$ & $E$. & . & . \\
\hline F16 & $\mathrm{G}$ & A. & . $A$ & . & . & . $\mathrm{T}$ & . & . & . & . & . & . & $A$ & . & . & C & $\mathrm{T}$ & & . & $A$ & $A$ &. & $\mathrm{G} \mathrm{G}$ & $\mathrm{GC}$ & {$[$.} & . & . \\
\hline F17 & G & A. & . $A$ & . & . & . & . & . & . & . & . & . &. & . & . & . & & & . & C & $A$ & $\mathrm{CC}$ & G G & $\mathrm{GC}$ & {$[$.} & . & . \\
\hline F18 & G & . $\mathrm{A}$ & A. & . & . & . . & . & . & . & . & . & . & . & . & . & & & & . & C & $A$ & $C$ & G G & $\mathrm{G} C$ & {$[$.} & . & . \\
\hline F19 & . & . . & . & . & T & $\mathrm{T} T$ & - & - & - & - & - & - & A & A & $\mathrm{T}$ & . & & & . & C & $A$ & $\mathrm{CC}$ & G G & $\mathrm{GCC}$ & $E$. & . & . \\
\hline$F 20$ & $\mathrm{G}$ & . $A$ & A. & . & . & . . & . & . & . & . & . & . & . & . & $\mathrm{T}$ & & & & . & C & $A$ & $\mathrm{CC}$ & G G & $\mathrm{GCC}$ & {$[$.} & . & . \\
\hline$C 1$ & . & . & . & . & T & $\mathrm{T} T$ & - & - & - & - & - & - & $A$ & $A$ & $\mathrm{~T}$ & . & & & . & C & $A$ & . & . . & . - & G & A & A \\
\hline$C 2$ & . & . & . $A$ & . & $\mathrm{T}$ & $\mathrm{T} T$ & - & - & - & - & - & - & $A$ & A & $\mathrm{T}$ & . & r. & & . & . & . & . & . & . - & - G & A & A \\
\hline$C 3$ & G & . & . $A$ & . & . & . . & . & . & 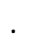 & . & $\cdot$ & $\cdot$ & . & - & . & . & & & . & . & . & . & . & - & G & A & $A$ \\
\hline C4 & . & . & . $A$ & C T & $\mathrm{T} \quad \mathrm{T}$ & $\mathrm{T} T$ & . & . & . & . & . & . & A & & & & & & . & A & $A$ & & G. & - & - $G$ & A & $A$ \\
\hline$C 5$ & . & . & . $A$ & $C T$ & $\mathrm{~T}$. & . $\mathrm{T}$ & . & . & . & . & . & . & A & & & & T & & . & $A$ & $A$ & $c$ & G. & - & G & A & $A$ \\
\hline C6 & G & A. & . $A$ & . & . & . $T$ & . & . & . & . & . & . & $A$ & 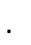 & & C & T & & . & A & $A$ & $c$ & G. & - & G & A & A \\
\hline$C 7$ & . & . & . $A$ & C T & $\mathrm{T} \quad \mathrm{T}$ & $\mathrm{T} T$ & . & . & . & $\cdot$ & $\cdot$ & . & A & & & $c$ & & & . & A & $A$ & . & G. & - & G & A & A \\
\hline$C 8$ & G & . & . $A$ & . . & . $\mathrm{T}$ & $\mathrm{T} T$ & . & . & . & . & - & . & A & & & & & & . & A & $A$ & & G & . - & G & A & $A$ \\
\hline$C 9$ & G & A. & . $A$ & . & . & . . . & . & . & . & . & . & . & & & & & & & . & A & $A$ & & G. & . - & G & $A$ & $A$ \\
\hline C10 & $\mathrm{G}$ & . & . $A$ & . & . & . & . & . & . & . & . & . & & & & & & & . & A & $A$ & & G. & . - & G & $A$ & $A$ \\
\hline C11 & G & . $\mathrm{A}$ & $A$ & . & . & . . & . & & & & & & & & & & & & . & C & $A$ & & $\mathrm{G}$ & - & & & $A$ \\
\hline C12 & & & . $A$ & . . & . $\mathrm{T}$ & & & & & & & & & & & & & & & & & & G. & & & A & \\
\hline
\end{tabular}

Fig. 1 The 32 eba-175 alleles detected from 131 Thai patients infected with malaria. Dots indicate identity with allele C1 nucleotide sequence. Dashes indicate insertions/deletions. F and C segments are not shown.

\section{Estimated divergence time}

The $d_{\mathrm{s}}$ values for regions II and III for all the pairs formed by the 32 distinct eba-175 alleles were calculated to estimate tMRCA of $e b a-175$ alleles in the Thai population. The neutral mutation rate of the $e b a-175$ gene was extrapolated from the calibration point derived from the divergence time of $P$. falciparum and $P$. reichenowi (6 MYA) $[5,20]$ by using the linearized tree of $e b a-175$ alleles in the MEGA program [13]. Thus, the estimated neutral mutation rate was $1.0 \times 10^{-8}$ per site per year.
Accordingly, the estimated tMRCA for eba-175 was approximately 0.14 MYA. The number of synonymous substitutions observed in Thai $P$. falciparum isolates was small (maximum $d_{\mathrm{S}}$ value $=2$ ); consequently, MCMCTree was used to estimate the tMRCA of $e b a-175$ alleles based on amino acid substitutions within region III. In this case, the estimated tMRCA was approximately 0.13 MYA (95\% highest posterior density confidential interval $=0.07-0.23$ MYA). Notably, these estimations rely 


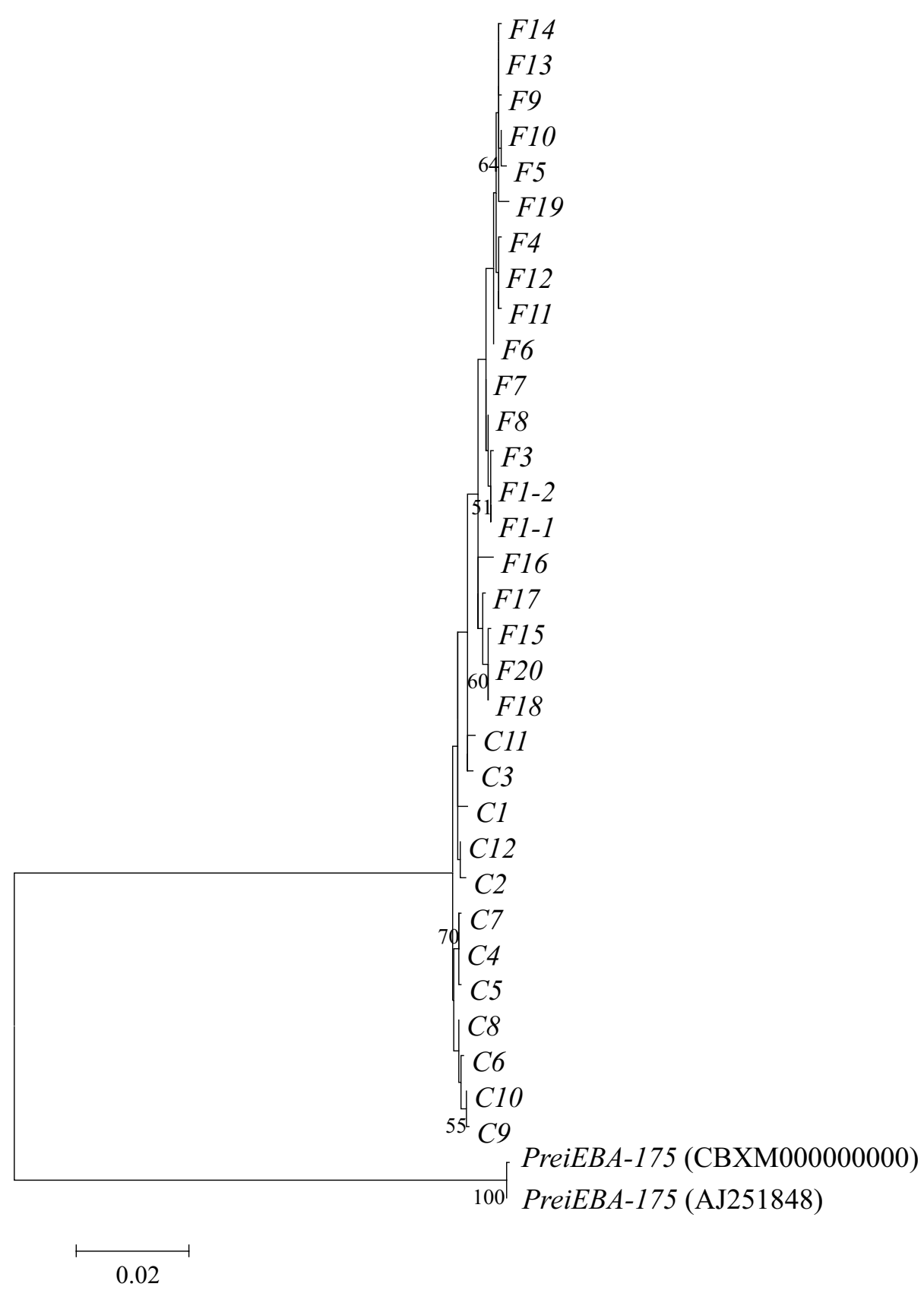

Fig. 2 Maximum likelihood tree of eba-175 (2,304 bp) regions II and III from Thai Plasmodium falciparum. The distance based on nucleotide substitutions was calculated by the Hasegawa-Kishino-Yano model. Two P. reichenowi eba-175-like (Prei_eba-175) sequences were used as outgroups. Only bootstrap values larger than $50 \%$ are shown.

on the assumption that $P$. falciparum and $P$. reichenowi diverged 6 MYA.

\section{Diversifying selection on eba-175 region II}

To search a signal of natural selection on region II of eba-175 in Thai P. falciparum, the $d_{\mathrm{N}}$ and $d_{\mathrm{S}}$ values for 32 long sequences $(2,310 \mathrm{bp})$ were calculated (Table 1$)$.
A comparison of the two values revealed that the $d_{\mathrm{N}}$ was significantly larger than $d_{\mathrm{S}}\left(P\right.$ value $<2.2 \times 10^{-16}$, Wilcoxon signed-rank test), suggesting that diversifying selection has influenced $e b a-175$ diversification in human P. falciparum. In addition, this result was further supported by the comparison between $\pi_{\mathrm{N}}$ and $\pi_{\mathrm{S}}$, including the allele frequencies of 131 Thai $P$. falciparum 


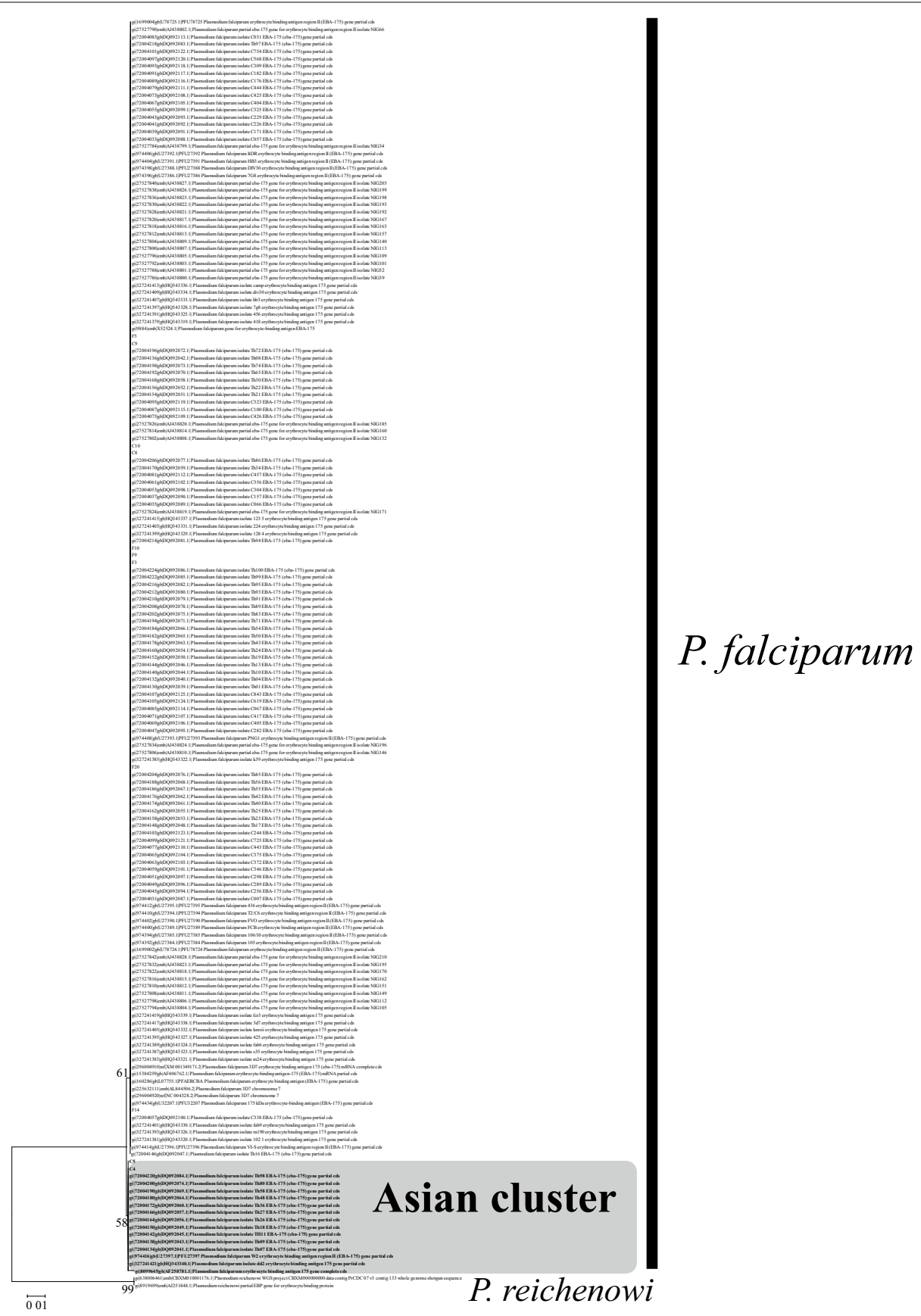

Fig. 3 Neighbor-joining tree of eba-175 partial region II (1,692 bp) of P. falciparum across the world. The distance based on synonymous substitutions was calculated using the Nei-Gojobori method with Jukes-Cantor model. Two P. reichenowi eba-175-like sequences were used as outgroups. Only bootstrap values larger than 50\% are shown.

isolates $\left(\pi_{\mathrm{N}} / \pi_{\mathrm{S}}=2.62 ; P\right.$ value $<2.2 \times 10^{-16}$, Wilcoxon signed-rank test). Subsequently, it was examined whether diversifying selection has also operated within other taxa, using sequence data from 16 and 11 Plasmodium spp. infecting chimpanzees and gorillas, respectively, and four $P$. reichenowi isolates (Table 1 ). Long sequence data from
African apes Plasmodium isolates were unavailable; consequently, region II short sequences (390-396 bp) were used for this analysis. Similar to the results using long sequences, $d_{\mathrm{N}}$ was significantly larger than $d_{\mathrm{S}}$ in human $P$. falciparum ( $P$ value $<2.2 \times 10^{-16}$, Wilcoxon signedrank test); on the other hand, $d_{\mathrm{N}}$ was significantly smaller 
Table 1 Comparison of nonsynonymous $\left(d_{N}\right)$ and synonymous ( $d_{S}$ substitution rates in Plasmodium eba-175 region II)

\begin{tabular}{|c|c|c|c|c|c|c|}
\hline Species & $N$ & Length (bp) & $d_{\mathrm{N}}$ & $d_{\mathrm{S}}$ & $d_{\mathrm{N}} / d_{\mathrm{S}}$ & $P$ value \\
\hline \multirow[t]{2}{*}{ Human Plasmodium falciparum isolates } & 32 & 2,310 & $0.005 \pm 0.001$ & $0.002 \pm 0.001$ & 2.98 & $<2.2 \times 10^{-16}$ \\
\hline & 32 & 390 & $0.010 \pm 0.004$ & $0.000 \pm 0.000$ & $N / A$ & $\mathrm{~N} / \mathrm{A}$ \\
\hline Chimpanzee Plasmodium isolates & 16 & 396 & $0.089 \pm 0.013$ & $0.110 \pm 0.026$ & 0.81 & $<2.2 \times 10^{-16}$ \\
\hline Gorilla Plasmodium isolates & 11 & 396 & $0.169 \pm 0.019$ & $0.207 \pm 0.037$ & 0.82 & $<2.2 \times 10^{-16}$ \\
\hline Plasmodium reichenowi & 5 & 393 & $0.003 \pm 0.002$ & $0.005 \pm 0.005$ & 0.47 & 0.32 \\
\hline
\end{tabular}

N/A not applicable.

than $d_{\mathrm{S}}$ in African apes Plasmodium (each $P$ value $<2.2 \times 10^{-16}$, Wilcoxon signed-rank test for chimpanzee and gorilla Plasmodium isolates). No significant differences between $d_{\mathrm{N}}$ and $d_{\mathrm{S}}$ were detected for $P$. reichenowi $(P$ value $=0.32$, Wilcoxon signed-rank test $)$. These results suggest that diversifying selection has only acted on $e b a$ 175 in human P. falciparum.

The McDonald-Kreitman (MK) test [23] for sequences of eba-175 region II in 32 Thai $P$. falciparum alleles and one in $P$. reichenowi showed that the ratio of nonsynonymous to synonymous polymorphic sites within species $(P \mathrm{n} / P \mathrm{~s}=19 / 1)$ was significantly higher than that of fixed sites between species $(D \mathrm{n} / D \mathrm{~s}=116 / 48 ; P=0.017$, Fisher's exact test). However, the MK test for Plasmodium isolates from African apes did not show significant differences between $P \mathrm{n} / P_{\mathrm{s}}$ and $D \mathrm{n} / D \mathrm{~s}\left(P \mathrm{n} / P_{\mathrm{s}}=59 / 23\right.$ and $D \mathrm{n} / D \mathrm{~s}=14 / 6, P$-value $=1.00$ for 16 chimpanzee Plasmodium isolates and one $P$. reichenowi; $P \mathrm{n} / P \mathrm{~s}=81 / 29$ and $D \mathrm{n} / D \mathrm{~s}=6 / 4, P=0.46$ for 11 gorilla Plasmodium isolates and one $P$. reichenowi). The results from the MK test suggest that either purifying selection or diversifying selection has acted on $e b a-175$ in Thai $P$. falciparum population.

Tajima's $D$ test [25] was used to test for departure from selective neutrality in $131 \mathrm{eba}-175$ sequences of Thai $P$. falciparum. This test compares two population genetic parameters, one estimated from mean pairwise nucleotide differences and the other from the number of mutations. The results revealed a significant positive Tajima's $D$ value (Tajima's $D$ statistic $=3.01$ and $P$ value $<0.01$ ), suggesting the balancing selection on $e b a-175$ in Thai $P$. falciparum. The possibility of a recent reduction in population size that provided the significant positive Tajima's $D$ statistic may be excluded, since both the $d_{\mathrm{N}} / d_{\mathrm{S}}$ ratio test and the MK test also suggested the existence of positive diversifying selection.

To assess the relation between amino acid variability and the erythrocyte binding site, the level of amino acid variability at each site was examined using a Wu-Kabat plot (Additional file 2). The Wu-Kabat plot showed that the level of amino acid variability at some codon sites outside the sites of direct interaction between $P$. falciparum EBA-175 and human GYPA molecules [28] was higher than the variation level at their interaction sites. These variable sites may be located in regions recognized by human antibodies if the higher degree of variation is caused by diversifying selection favoring mutations to other amino acids.

\section{Discussion}

Modern humans are believed to have emerged in Africa approximately 0.2 MYA and subsequently dispersed and colonized other continents after their exodus from Africa (known as the "out-of-Africa event") approximately 0.1 MYA, although the accuracy of these estimates remains a contentious issue [29-32]. Here tMRCA for Thai $P$. falciparum eba-175 was examined, and our results, 0.14 MYA from synonymous substitutions and 0.13 MYA from amino acid substitutions, suggest that the tMRCA of Thai P. falciparum may predate the out-ofAfrica event but after the emergence of modern humans. However, this estimation relies largely on the assumption that $P$. falciparum diverged from $P$. reichenowi 6 MYA $[5,20]$. A recent phylogenetic analysis for Plasmodium infecting Homininae species suggested that Plasmodium infecting gorillas was transmitted to modern humans in recent times [33]. This possible recent evolutionary origin of human $P$. falciparum is incompatible with the present assumption of that $P$. falciparum $/ P$. reichenowi divergence time is similar to that of humans/chimpanzees (i.e., ca. 6 MYA [34-37]). However, regardless of the tMRCA estimate, ancestral human dispersal and recent increase in human population size, resulting in the increase in $P$. falciparum population size, are likely to have contributed to the eba-175 diversification of $P$. falciparum (i.e., the emergence of Asian-specific clade).

The comparison of $d_{\mathrm{N}} / d_{\mathrm{S}}$ ratios of $e b a-175$ region II sequences among Plasmodium species showed an excess of nonsynonymous substitutions over synonymous substitutions in human P. falciparum but not in Plasmodium spp. infecting African apes and $P$. reichenowi. The result suggests that diversifying selection has affected $e b a-175$ region II in Thai $P$. falciparum, as previously reported [8-11]. The results on $\pi_{N} / \pi_{S}$ ratios and MK test [23] also supported the diversifying selection hypothesis. On the other hand, the estimated $d_{N} / d_{S}$ ratios and MK test [23] 
suggested that eba-175 genetic diversities in other Plasmodium relatives have not been affected by diversifying selection. Thus, the EBA-175 protein may be the target for the immune response against $P$. falciparum malaria only in humans.

In this study, the tMRCA was estimated under the assumption of no inter-allelic recombination. The presence of inter-allelic recombination may affect the estimation of tMRCA. Since the DnaSP program [24] suggested the existence of recombination among 32 eba-175 alleles (Minimum number of recombination events, Rm: 8), we investigated the possible recombinants by using the GENECONV program [38], and detected 21 possible recombinants. After the removal of 21 possible recombinants, we recalculated the tMRCA of the remaining 11 eba-175 alleles in Thai P. falciparum. The estimated tMRCA was approximately 0.12 MYA that was roughly equivalent to the tMRCA (0.14 MYA) estimated by using all of 32 alleles. In addition, the $d_{\mathrm{N}} / d_{\mathrm{S}}$ ratio of 11 sequences not including recombinant sequences (2.46) was not largely different from that of 32 sequences (2.98). Thus, our results seem not to be affected by the presence of possible recombinants.

\section{Conclusions}

The genetic diversity in region II of P. falciparum eba175 seems to have been increased after the exodus from Africa of ancestral modern humans. As a consequence of the human expansion followed by an increase in $P$. falciparum population size, diversifying selection may have efficiently maintained the nonsynonymous substitutions in region II of Thai P. falciparum eba-175. Human dispersal out of Africa would have had a major impact on molecular evolution of P. falciparum eba-175.

\section{Additional files}

\section{Additional file 1: Neighbor-net network of eba-175 alleles from a varied populations worldwide. The network was constructed based on synony- mous substitutions of the partial region II from Plasmodium falciparum. Two $P$. reichenowi eba-175 sequences were used as outgroups. \\ Additional file 2: Level of variability for amino acid residues among eba-175 alleles estimated using a Wu-Kabat plot. Amino acid sequences from regions II and III of eba-175 alleles were used to construct a Wu- Kabat plot. The ordinate axis represents the level of amino acid variability. The abscissa axis represents the amino acid position. Pink bars indicate residues involved in the interaction with human GYPA molecules [28].}

\section{Authors' contributions}

YY conducted the statistical analyses and wrote the manuscript. JP and $\mathrm{HH}$ collected blood samples. IN, JP, and HH extracted DNA from blood samples. IN carried out the experiments. YY, JP, and JO participated in the design of the study. JO was involved in the interpretation of the data and preparation of the manuscript. All authors have read and approved the final manuscript.

\section{Author details}

Department of Biological Sciences, Graduate School of Science, The University of Tokyo, 7-3-1, Hongo, Bunkyo-ku, Tokyo 113-0033, Japan. ${ }^{2}$ Department of Human Genetics, Graduate School of Medicine, The University of Tokyo, Tokyo, Japan. ${ }^{3}$ Department of Microbiology and Immunology, Faculty of Tropical Medicine, Mahidol University, Bangkok, Thailand. ${ }^{4}$ Department of Social and Environmental Medicine, Faculty of Tropical Medicine, Mahidol University, Bangkok, Thailand.

\section{Acknowledgements}

The authors deeply thank all the subjects for their participation in the study. This study was partly supported by KAKENHI (25650125) Grant-in-Aid for Challenging Exploratory Research and KAKENHI (26305015) Grant-in-Aid for Scientific Research (B), the Takeda Science Foundation, and KAKENHI (26-1601) Grant-in-Aid for JSPS Fellows.

\section{Compliance with ethical guidelines}

\section{Competing interests}

The authors declare that they have no competing interests.

Received: 27 January 2015 Accepted: 21 May 2015

Published online: 01 August 2015

\section{References}

1. Rich SM, Ayala FJ (2006) Evolutionary origins of human malaria parasites. In: Arese P, Dronamraju KR (eds) Malar Genet Evol Asp. Springer, New York, pp 125-146 (Emerging Infectious Diseases of the 21st Century)

2. Sutherland CJ, Tanomsing N, Nolder D, Oguike M, Jennison C, Pukrittayakamee $S$ et al (2010) Two nonrecombining sympatric forms of the human malaria parasite Plasmodium ovale occur globally. J Infect Dis 201:1544-1550

3. Vargas-Serrato E, Corredor V, Galinski MR (2003) Phylogenetic analysis of CSP and MSP-9 gene sequences demonstrates the close relationship of Plasmodium coatneyi to Plasmodium knowlesi. Infect Genet Evol 3:67-73

4. Martinsen ES, Perkins SL, Schall JJ (2008) A three-genome phylogeny of malaria parasites (Plasmodium and closely related genera): evolution of life-history traits and host switches. Mol Phylogenet Evol 47:261-273

5. Prugnolle F, Durand P, Ollomo B, Duval L, Ariey F, Arnathau C et al (2011) A fresh look at the origin of Plasmodium falciparum, the most malignant malaria agent. PLoS Pathog 7:e1001283

6. Adams JH, Sim BK, Dolan SA, Fang X, Kaslow DC, Miller LH (1992) A family of erythrocyte binding proteins of malaria parasites. Proc Natl Acad Sci 89:7085-7089

7. Sim B, Chitnis C, Wasniowska K, Hadley T, Miller L (1994) Receptor and ligand domains for invasion of erythrocytes by Plasmodium falciparum. Science 264:1941-1944

8. Baum J, Thomas AW, Conway DJ (2003) Evidence for diversifying selection on erythrocyte-binding antigens of Plasmodium falciparum and $P$. vivax. Genetics 163:1327-1336

9. Escalante A, Lal A, Ayala F (1998) Genetic polymorphism and natural selection in the malaria parasite Plasmodium falciparum. Genetics 149:189-202

10. Wang HY, Tang H, Shen CKJ, Wu Cl (2003) Rapidly evolving genes in human. I. The glycophorins and their possible role in evading malaria parasites. Mol Biol Evol 20:1795-1804

11. Verra F, Chokejindachai W, Weedall GD, Polley SD, Mwangi TW, Marsh K et al (2006) Contrasting signatures of selection on the Plasmodium falciparum erythrocyte binding antigen gene family. Mol Biochem Parasitol 149:182-190

12. Benson DA, Cavanaugh M, Clark K, Karsch-Mizrachi I, Lipman DJ, Ostell J et al (2013) GenBank. Nucleic Acids Res 41:D36-D42

13. Tamura K, Peterson D, Peterson N, Stecher G, Nei M, Kumar S (2011) MEGA5: molecular evolutionary genetics analysis using maximum likelihood, evolutionary distance, and maximum parsimony methods. Mol Biol Evol 28:2731-2739 
14. Hasegawa M, Kishino H, Yano T (1985) Dating of the human-ape splitting by a molecular clock of mitochondrial DNA. J Mol Evol 22:160-174

15. Saitou N, Nei M (1987) The neighbor-joining method: a new method for reconstructing phylogenetic trees. Mol Biol Evol 4:406-425

16. Nei M, Gojobori T (1986) Simple methods for estimating the numbers of synonymous and nonsynonymous nucleotide substitutions. Mol Biol Evol 3:418-426

17. Jukes T, Cantor C (1969) Evolution of protein molecules. In: Munro H (ed) Mamm protein Metab. Academic Press, New York, pp 21-132

18. Bryant D, Moulton V (2004) Neighbor-net: an agglomerative method for the construction of phylogenetic networks. Mol Biol Evol 21:255-265

19. Huson DH, Bryant D (2006) Application of phylogenetic networks in evolutionary studies. Mol Biol Evol 23:254-267

20. Escalante AA, Ayala FJ (1994) Phylogeny of the malarial genus Plasmodium, derived from rRNA gene sequences. Proc Natl Acad Sci 91:11373-11377

21. Yang Z (2007) PAML 4: phylogenetic analysis by maximum likelihood. Mol Biol Evol 24:1586-1591

22. Whelan S, Goldman N (2001) A general empirical model of protein evolution derived from multiple protein families using a maximum-likelihood approach. Mol Biol Evol 18:691-699

23. McDonald JH, Kreitman M (1991) Adaptive protein evolution at the Adh locus in Drosophila. Nature 351:652-654

24. Librado P, Rozas J (2009) DnaSP v5: a software for comprehensive analysis of DNA polymorphism data. Bioinformatics 25:1451-1452

25. Tajima F (1989) Statistical method for testing the neutral mutation hypothesis by DNA polymorphism. Genetics 123:585-595

26. Wu T, Kabat E (1970) An analysis of the sequences of the variable regions of Bence Jones proteins and myeloma light chains and their implications for antibody complementarity. J Exp Med 132:211-250
27. Ware LA, Kain KC, Lee Sim BK, Haynes JD, Baird JK, Lanar DE (1993) Two alleles of the 175-kilodalton Plasmodium falciparum erythrocyte binding antigen. Mol Biochem Parasitol 60:105-109

28. Tolia NH, Enemark EJ, Sim BKL, Joshua-Tor L (2005) Structural basis for the EBA-175 erythrocyte invasion pathway of the malaria parasite Plasmodium falciparum. Cell 122:183-193

29. Excoffier $L$ (2002) Human demographic history: refining the recent African origin model. Curr Opin Genet Dev 12:675-682

30. Stringer C (2002) Modern human origins: progress and prospects. Philos Trans R Soc Lond B Biol Sci 357:563-579

31. Stringer C (2003) Human evolution: out of Ethiopia. Nature 423:692-695

32. Stewart JR, Stringer CB (2012) Human evolution out of Africa: the role of refugia and climate change. Science 335:1317-1321

33. Liu W, Li Y, Learn GH, Rudicell RS, Robertson JD, Keele BF et al (2010) Origin of the human malaria parasite Plasmodium falciparum in gorillas. Nature 467:420-425

34. Satta Y, Hickerson M, Watanabe H, O'hUigi C, Klein J (2004) Ancestral population sizes and species divergence times in the primate lineage on the basis of intron and BAC end sequences. J Mol Evol 59:478-487

35. Steiper ME, Young NM (2006) Primate molecular divergence dates. Mol Phylogenet Evol 41:384-394

36. Matsui A, Hasegawa M (2012) Molecular phylogeny and evolution in primates. In: Imai H, Go Y, Hirai H (eds) Post-Genome Biol Primates. Springer Tokyo, Tokyo, pp 243-267 (Primatology Monographs)

37. Scally A, Dutheil JY, Hillier LW, Jordan GE, Goodhead I, Herrero J et al (2012) Insights into hominid evolution from the gorilla genome sequence. Nature 483:169-175

38. Sawyer S (1989) Statistical tests for detecting gene conversion. Mol Biol Evol 6:526-538

\section{Submit your next manuscript to BioMed Central and take full advantage of:}

- Convenient online submission

- Thorough peer review

- No space constraints or color figure charges

- Immediate publication on acceptance

- Inclusion in PubMed, CAS, Scopus and Google Scholar

- Research which is freely available for redistribution

Submit your manuscript at

www.biomedcentral.com/submit

C BioMed Central 